\title{
Editorial: Biological Psychology/Pharmacopsychology
}

The section Biological Psychology/Pharmacopsychol-ogy has been designed to cover work on the relationship between biochemical processes and behaviour of normal subjects and animals. Investigations based on clinical samples will be included if psychological measurement devices or psychological experimental techniques are applied.

During the last years it has become quite clear that investigations on the relationship between biochemical and behavioural processes using normal subjects or animals provide very important data for understanding behaviour and behavioural disorders. Thus, the section Biological Psychology/Pharmacopsychology will give valuable information not only to psychologists, behavioural pharmacologists and biochemists but as well to clinical scientists, e.g., psychiatrists.

The relationship between biochemical and behavioural processes can be explored by two basic strategies which will be systematically treated in this section. The first research strategy uses biochemical parameters as dependent variables for studying the effects of behavioural or situational manipulations or variations. This strategy has become quite powerful during the last years by the invention or improvement of general biochemical and neurochemical indicator methods, e.g., glucose uptake of the brain or enzymes involved in the metabolism of neurotransmitters.

This section will publish papers which will deal with all important psychological functions (perception and thinking, emotion and motivation, memory, psychomo-tor behaviour, social interaction) and biochemical/neuro-chemical systems. In the next volumes of Neuropsychobi- 
ology a series of review papers will deal with the most important constructs used in normal psychology and pa-thopsychology.

The second research strategy uses behavioural parameters (e.g., performance tests, scaling of anxiety) as dependent variables for studying the effects of biochemical manipulations which are mostly achieved by the administration of chemical compounds, e.g., drugs or hormones.

A major object of the section Biological Psychology/Pharmacopsychology is concerned with the influence of chemical substances (drugs, hormones, neuromodula-tors and environmental chemical compounds) on behaviour. This section will publish papers treating all types of chemical compounds which influence the behaviour of normal subjects and animals. Moreover, review papers will elucidate how various behavioural functions may be studied by the administration of chemical compounds which serve as research tools.

The section Biological Psychology/Pharmacopsychology will put special emphasis on essential problems and research methodology by means of review and theoretical papers. Regularly, papers devoted to new methods of data collection, on experimental design and statistical evaluation will be published.

Another series of papers will review the usefulness of experiments with healthy subjects and animals for various fields of biological psychiary, e.g., for psychophar-macotherapy. This series points to one of the major and unique aims of Neuropsychobiology, the co-operation between the various neurosciences and biosciences. 